\title{
910 OVEREXPRESSION OF MIR-155-5P CAN UPREGULATE ANTIGEN PROCESSING AND PRESENTATION PATHWAY VIA TARGETING TAPASIN
}

Yuan Wang ${ }^{*}$, Maria-Filothei Lazaridou, Chiara Massa, Barbara Seliger. Institute of Medical Immunology, Halle (Saale), Germany

Background Dysregulation of major histocompatibility complex (MHC) class I antigen processing and presentation machinery (APM) components in the tumor as one main molecular mechanism of immune escape leading to deactivation of $\mathrm{T}$ cell immune surveillance could be due to post-transcriptional regulation via immune-modulatory microRNAs (miRNA). It is now well established from a variety of studies that several miRNAs could effectively modulate the expression of some MHC class I APM components in tumors. Tapasin is an important APM molecule involved in the association of MHC class I with transporter associated with antigen processing (TAP) and peptide loading. Since so far no detailed investigation of the posttranscriptional regulation of tapasin exists, the aim of this study is to identify and functionally characterize miRNAs targeting tapasin in melanoma.

Methods Using miRNA trapping by RNA in vitro affinity purification (miTRAP) and in silico as well as small RNA sequencing, miRNAs will be identified, which bind to the 3'untranslated region (3' UTR) of tapasin. Dual-luciferase assays will be performed to determine to bind of the miRNA. In silico analysis was performed to predict the effect of miRNAs on the survival of melanoma patients in correlation to tapasin. RT-qPCR, Western blot, flow cytometry, and other functional assays were performed after transfecting miRNA mimics in three melanoma cell lines.

Results Using the combination strategy of miTRAP and RNA seq we identified miR-155-5p to bind to the 3'UTR of tapasin, which was further confirmed by in silico analysis and dual-luciferase reporter assay. Transfection of miR-155-5p mimics demonstrated that miR-155-5p upregulate tapasin protein level, which was accompanied by an upregulation of the MHC class I (HLA-ABC) surface expression. Simultaneously, in several different types of cancer, including melanoma, the expression of miR-155-5p is significantly positively correlated with the patient's survival and HLA-A protein.

Conclusions Our data revealed for the first time a positive role of miR-155-5p in the posttranscriptional regulation of tapasin in melanoma and provide further insights into the miR-155-5p-mediated induction of HLA-ABC surface expression. This might lead to a better $\mathrm{T}$ cell response, avoidance tumor cell escape, improvement of patients' survival and thus might be a potential therapeutic target.

Acknowledgements The work was supported by a grant from the DKH (BS).

http://dx.doi.org/10.1136/jitc-2021-SITC2021.910 